\title{
Parallel optical control of spatiotemporal neuronal spike activity using high-speed digital light processing
}

\section{Jason Jerome' ${ }^{1}$, Robert C. Foehring ${ }^{1}$, William E. Armstrong ${ }^{1}$, William J. Spain ${ }^{2,3}$ and Detlef H. Heck ${ }^{1 *}$}

1 Department of Anatomy and Neurobiology, University of Tennessee Health Science Center, Memphis, TN, USA

2 Neurology Section, Veterans Affairs Puget Sound Health Care System, Seattle, WA, USA

${ }^{3}$ Department of Physiology and Biophysics, University of Washington, Seattle, WA, USA

\section{Edited by:}

Neeraj Jain, National Brain Research

Centre, India

\section{Reviewed by:}

Upinder S. Bhalla, National Center for Biological Sciences, India

Christine E. Collins, Vanderbilt

University, USA

Gregor Rainer, University of Fribourg,

Switzerland

\section{${ }^{*}$ Correspondence:}

Detlef H. Heck, Department of

Anatomy and Neurobiology, University of Tennessee Health Science Center, 855 Monroe Avenue, Suite 515, Memphis, TN 38163, USA.

e-mail:dheck@uthsc.edu
Neurons in the mammalian neocortex receive inputs from and communicate back to thousands of other neurons, creating complex spatiotemporal activity patterns. The experimental investigation of these parallel dynamic interactions has been limited due to the technical challenges of monitoring or manipulating neuronal activity at that level of complexity. Here we describe a new massively parallel photostimulation system that can be used to control action potential firing in in vitro brain slices with high spatial and temporal resolution while performing extracellular or intracellular electrophysiological measurements. The system uses digital light processing technology to generate 2-dimensional (2D) stimulus patterns with $>780,000$ independently controlled photostimulation sites that operate at high spatial $(5.4 \mu \mathrm{m})$ and temporal $(>13 \mathrm{kHz})$ resolution. Light is projected through the quartz-glass bottom of the perfusion chamber providing access to a large area $(2.76 \mathrm{~mm} \times 2.07 \mathrm{~mm})$ of the slice preparation. This system has the unique capability to induce temporally precise action potential firing in large groups of neurons distributed over a wide area covering several cortical columns. Parallel photostimulation opens up new opportunities for the in vitro experimental investigation of spatiotemporal neuronal interactions at a broad range of anatomical scales.

Keywords: parallel photostimulation, digital light processing, caged glutamate, neuronal synchrony, barrel cortex

\section{INTRODUCTION}

Understanding neuronal information processing and neuronal communication in highly interconnected neuronal networks is one of the great challenges of neuroscience. Changes in the rate of action potential firing in individual neurons have been shown to represent specific features of sensory stimuli and motor commands (Hubel and Wiesel, 1968; Georgopoulos et al., 1984). More recently, parallel recordings from multiple neurons suggests that sensory and motor information are also represented by groups of neurons generating synchronous, oscillatory, or more complex spatiotemporal patterns of action potentials (Vaadia et al., 1995; Kreiter and Singer, 1996; Riehle et al., 1997; Prut et al., 1998; Steinmetz et al., 2000; Huetz et al., 2009; Gutierrez et al., 2010). However, we have only a very poor understanding of the neurophysiological processes that lead to the generation of such precisely coordinated activity patterns. Current experimental techniques do not allow the precise spatiotemporal manipulation of spiking activity in large groups of neurons, which would allow a systematic analysis of the conditions sufficient and necessary to generate and propagate such patterns. Consequently, most of our current knowledge about the generation and propagation of synchronous or otherwise coordinated population spike patterns in neocortex-like networks comes from theoretical and simulation studies (Salinas and Sejnowski, 2001; Kumar et al., 2010).

Techniques involving the photolytic release of caged neurotransmitters in acute slices provide unique opportunities for manipulating neuronal activity with high spatial and temporal resolution. In these techniques, the caged neurotransmitter is inactive until it is photolytically released with ultraviolet light in a fast single-photon process. However, because most current systems use a single photostimulation beam, induction of truly synchronous activity in neurons at thousands of anatomically distributed sites as it occurs in vivo is not feasible but can only be approximated with rapid sequential stimulation (Boucsein et al., 2005).

Here, we describe a massively parallel photostimulation system that provides new opportunities for the experimental investigation of spatiotemporal neuronal interactions in living brain tissue through precise spatiotemporal control of spiking activity. The system uses digital light processing (DLP) technology to independently control $>780,000$ light beams with microsecond temporal and micron spatial resolution. With a large field of view $(2.7 \mathrm{~mm} \times 2 \mathrm{~mm})$ and high spatial resolution, when combined with photolytic neuronal activation this system provides precise optical control of supra- and sub-threshold neuronal activity at thousands of sites at a wide range of anatomical scales. Here we present a detailed description of the design and capabilities of this system and demonstrate its utility in investigating the propagation of synchronous population activity from cortical layer 4 to layer $2 / 3$ neurons.

\section{MATERIALS AND METHODS ACUTE SLICE PREPARATION}

All animal experimental procedures adhered to guidelines approved by the University of Tennessee Health Science Center Animal Care and Use Committee. Principles of laboratory animal care (NIH publication No. 86-23, rev. 1996) were followed. Young 
C57BL/6 (P14-P21) mice are housed within a breeding colony with 12-h light/dark cycles in standard cages with ad libitum access to food and water. For the preparation of brain slices mice were anesthetized in a 500-ml glass jar containing isofluranesoaked gauze and decapitated. The brain was quickly removed while the head was submerged in cold oxygen-bubbled dissection solution containing (in $\mathrm{mM}$ ): 250 sucrose, 15 HEPES, 11 glucose, $7 \mathrm{MgSO}_{4}, 3 \mathrm{KCl}, 1 \mathrm{NaH}_{2} \mathrm{PO}_{4}, 0.5 \mathrm{CaCl}_{2}$ ( $\mathrm{pH}: 7.3-7.4$ adjusted with $\mathrm{NaOH}, 300 \mathrm{mOsm} / \mathrm{l}$ ) with $250 \mathrm{mM}$ glycerol replacing sucrose in some experiments. $300 \mu \mathrm{m}$ coronal slices were made on a Vibratome 1500 (Vibratome, St. Louis) and then transferred to warm $\left(33^{\circ} \mathrm{C}\right) 95 \% \mathrm{O}_{2}-5 \% \mathrm{CO}_{2}$ - bubbled aCSF containing (in $\mathrm{mM}$ ): $125 \mathrm{NaCl}, 26 \mathrm{NaHCO}_{3}, 20$ glucose, $3 \mathrm{KCl}, 2 \mathrm{CaCl}_{2}, 2 \mathrm{MgCl}_{2}$, $1.25 \mathrm{NaH}_{2} \mathrm{PO}_{4}$ for $1 \mathrm{~h}$ before being transferred to a patch-clamp recording chamber.

\section{PATCH-CLAMP ELECTROPHYSIOLOGY}

Slices were transferred to a recording chamber and continually perfused with recirculated aCSF bubbled with 95\% $\mathrm{O}_{2}-5 \%$ $\mathrm{CO}_{2}$ and heated to $33^{\circ} \mathrm{C}$. Patch electrodes were fabricated from $1.50 \mathrm{~mm}$ OD $1.17 \mathrm{~mm}$ ID borosilicate glass to a tip resistance of 3-10 M $\Omega$ on a Flaming/Brown micropipette puller (P-97, Sutter Instruments Co., Novato, CA, USA) and filled with intracellular patch-solution containing (in $\mathrm{mM}$ ): $130.5 \mathrm{KMeSO}_{4}, 10 \mathrm{KCL}, 10$ EGTA, 10 HEPES, $7.5 \mathrm{NaCl}, 2 \mathrm{MgCl}_{2}$, 2 ATP, $0.5 \mathrm{GTP}$, plus, in some experiments, $1-3 \mathrm{mg} / \mathrm{ml}$ biocytin (Tocris). Pyramidal neurons in layers II/III and spiny neurons in layer IV of the somatosensory barrel cortex were visually identified using 40× Hoffmanmodulation Contrast optics on an Olympus BX50WI microscope and targeted for recordings. The cells were approached with slight positive pressure applied to the pipette solution. Once a visible dimple formed on the cell surface, light suction was applied to form a gigaohm seal, followed by a quick burst of suction to go into whole-cell mode. Membrane potential signals were amplified with an AxoClamp 2B patch-clamp amplifier, digitized with a Digidata 1322a, and stored to hard disk using pClamp 10 software (Molecular Devices, Sunnyvale, CA, USA). Junction potentials calculated with the Clampex junction potential tool $(-13.7 \mathrm{mV})$ were corrected offline for whole-cell recordings. $13.5 \mathrm{mg}$ "caged" MNI-glu trifluoroacetate (Femtonics Budapest, Hungary) was added to $12.5 \mathrm{ml}$ recirculating aCSF (2.5 mM MNI-glu in aCSF) for photostimulation.

Loose cell attached extracellular recordings used the same glass pipettes filled with (in $\mathrm{mM}$ ): $125 \mathrm{NaCl}, 2.5 \mathrm{KCl}, 2 \mathrm{CaCl}_{2}, 1 \mathrm{MgCl}_{2}$, and 10 HEPES (pH: 7.3-7.4 adjusted with $\mathrm{KOH}, 300 \mathrm{mOsm} / \mathrm{l}$ ). For these recordings, once a visible dimple formed on the cell surface, the positive pressure was released, and no suction was applied.

All measurements are reported as mean \pm SD.

\section{RECONSTRUCTION OF BIOCYTIN-FILLED CELLS}

After the recording, cells were stored overnight in fixative (PBS $+0.2 \%$ picric acid $+4 \%$ paraformaldehyde $)$. Cells were thoroughly rinsed with rinsing solution (PBS $+0.5 \%$ Triton $\mathrm{X}-100$ ) and then incubated with $2 \%$ Avidin-D fluorescein (Vector Laboratories, Burlingame, CA, USA) for 2-4 h. After another thorough rinsing, slices were mounted on coverslipped slides in 50\% glycerol-50\% PBS.

\section{DIGITAL MICROMIRROR DEVICE}

Uncaging of most photolabile compounds requires the use of ultraviolet light. We thus employed an ultraviolet-compatible version of the DLP Discovery 3000 kit (Digital Light Innovations, Austin, TX, USA). The digital micromirror device (DMD) communicates with a computer (Windows PC) via a USB interface board (ALP-3 High-Speed, Vialux, Germany). The USB kit includes a $\mathrm{CD}$ with a dynamic link library (DLL) containing $\mathrm{C}++$ functions for controlling the mirrors. The supporting documentation of this DLL describes the functions for loading sequences, starting and stopping sequences, and setting the timing of frame changes. A graphical-user-interface (GUI) for the generation of 2-dimensional stimulus patterns was programmed in C\# using Visual Studio 2008 Professional (Microsoft). USB control of the DLP system uses commands embedded in the ALP-3 DLL. Since these functions were written in $\mathrm{C}++$, the functions were imported and marshaled into their own wrapper class. Examples of C\# code for communicating with the DLP system are provided as Section "Appendix" and are also available upon request from the corresponding author.

The ALP-3 kit also includes enough RAM for buffering up to 1365 XGA $(1024 \times 768)$ binary (black/white) frames. Loading sequences to the RAM of the ALP-3 board required that sequences be represented as 3-dimensional byte arrays. To take advantage of the graphical capabilities of the Microsoft NET framework and C\#, photostimulation sequences are generated and graphical manipulations are performed on bitmap objects with .NET functions. The bitmaps are then converted, one at a time, into 3-dimensional byte arrays and loaded into the RAM of the ALP-3 board. The USB accessory board outputs a TTL signal with the "high" (5-volt) state indicating that a frame is being actively projected while the "down" (0-volt) state indicates either an interframe period or an idle DMD. The first high state pulse, i.e., the onset of the first frame of a stimulus sequence, was used to temporally align neuronal membrane potential recordings to the photostimulation sequences.

\section{OPTOMECHANICS}

The projection and illumination optics were assembled and aligned within a custom optical cage (60 and $30 \mathrm{~mm}$ cage systems, Thorlabs; additional parts machined by UTHSC biomedical instrumentation machine shop). In order for the DLP photostimulation optics to be incorporated into the electrophysiology rig for projection through the bottom of the perfusion chamber, the microscope's condenser, and the photostimulation optics had to be mounted onto precision sliding rails to position either the condenser or the DMD projection optics underneath the chamber. The condenser was positioned under the perfusion chamber while patch-clamp recordings from neurons were established under microscopic guidance. After a stable recording was established, the condenser was replaced by the projection optics. Start and end points of rail movements were precisely determined by adjustable mechanical stops. A simplified schematic and flow chart of this system is shown in Figure 1.

We illuminated the full area of the DMD with UV light using a 3-watt UV laser (frequency-tripled, q-switched, ND:YVO4, $355 \mathrm{~nm}$ laser, 3530-30, DPSS laser, Santa Clara, CA, USA). The beam was expanded and homogenized with a custom refractive beam shaper 


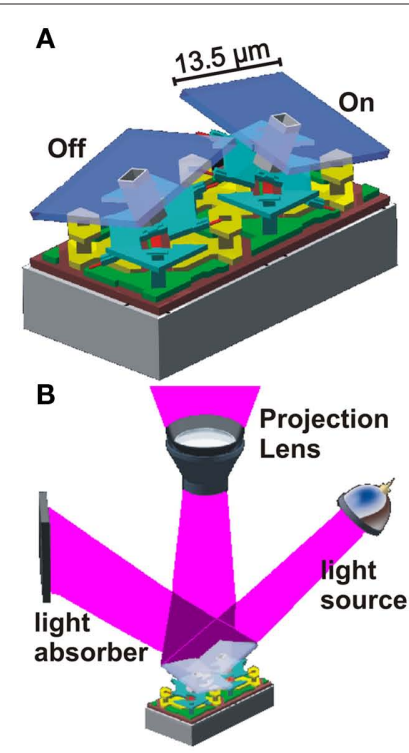

FIGURE 1 | Principal of digital micromirror device (DMD) function and integration of a DMD into the photostimulation light path. (A)Two DMD mirrors with one mirror (left) in the "off" and the other (right) in the "on" position (illustration adapted with permission from Texas Instruments). (B) Illustration of a two-mirror DLP system. Each micromirror reflects the light either toward the projection optics (left mirror in the bottom illustration) or toward a light absorber (right

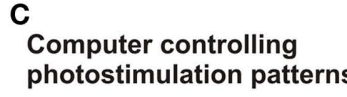

$$
\text { photostimulation patterns }
$$
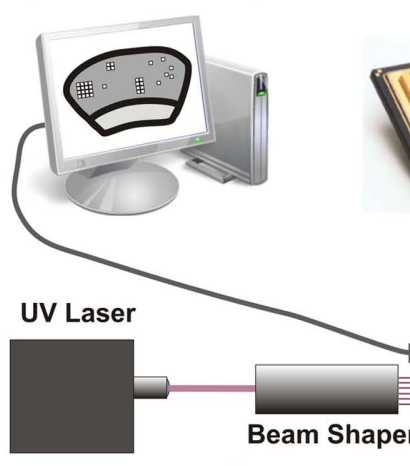

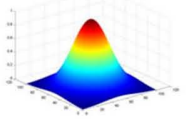

Gaussian (IN)
.
$-$

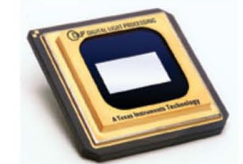

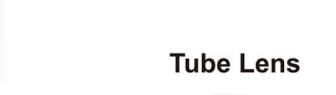
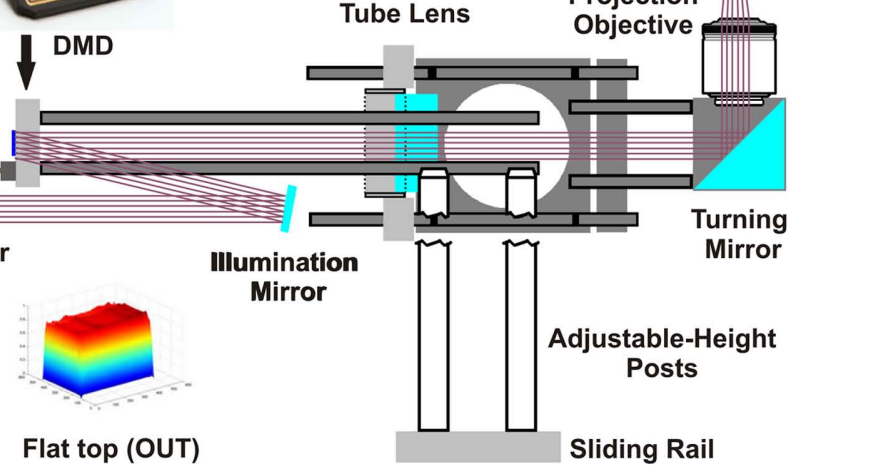

Projection

Objective

Flat top (OUT)

Sliding Rail

mirror; illustration adapted with permission from Texas Instruments). (C) Schematic drawing of the light path for the DLP photostimulation system using a UV laser, a "beam shaper" (Flat-Top Generator, StockerYale, NH) and the DMD to reflect UV light onto stimulation sites on the slice. The "beam shaper" alters the spatial intensity profile of the laser beam from Gaussian to a rectangular "flat-top" (color illustrations below beam shaper) to ensure homogeneous illumination of the DMD.
(StockerYale, Salem, NH, USA). The expanded homogenous beam was folded to the appropriate illumination angle with a high-power UV mirror (15087, Lambda Research Optics; Figure 1C). DMD micromirrors in the "off" position reflected light to a light trap consisting of a piece of aluminum painted with black permanent marker. Micromirrors in the "on" position reflected light orthogonal to the surface of the DMD through the projection optics to the perfusion chamber.

The projection optics consisted of a tube lens (P4033741038, Zeiss), which collimated the DMD-reflected light. The collimated light was then folded by a $90^{\circ}$ cube-mounted turning mirror (CM1-F01, Thorlabs). A high NA, low magnification microscope objective (Fluar 5×, NA 0.25, Carl Zeiss, Germany, "projection objective" in Figure 1C) focuses the image of the UV-illuminated On-positioned mirrors onto the neuronal tissue through a UV-permissive quartz-glass bottom (SPI supplies, 01015-AB, West Chester, PA, USA).

\section{RESULTS \\ SYSTEM DESIGN AND OPTICAL PERFORMANCE}

The key component of the DLP-based photostimulation system is the DMD (Texas Instruments). A DMD is a matrix of thousands of microscopic mirrors with each mirror serving as a single pixel in multimedia projectors and lithography systems. The mirrors have two tilt angles (Figure 1A). These "on" and "off" positions reflect light toward the projection target or toward a light trap, respectively (Figure 1B). Each mirror's tilt angle is independently controlled. Here we use the DMD to project 2-dimensional photostimulation patterns onto neuronal tissue. Multiple 2-dimensional frames are presented in sequence to generate spatiotemporal photostimulation patterns. The system allows a maximum frame rate of 13,333 frames per second.

Our design introduces photostimulation light via a separate optical path from beneath the perfusion chamber. This generates two major advantages over stimulation through the microscope objective: (1) It provides optical access to a much larger area of the slice and (2) it minimizes the uncaging of glutamate in the bath solution above the slice because photons are first absorbed within the slice. The projection objective demagnifies the image of the mirrors by a factor of 5, resulting in a projected pixel size of $2.7 \mu \mathrm{m} \times 2.7 \mu \mathrm{m}$. The objective generated a conical excitation profile with a high light-flux density at the focal plane within the brain slice and diffuse light above the slice. As configured, this system allows optical control of neuronal spike activity within a 2.76- $\mathrm{mm} \times 2.07-\mathrm{mm}$ area of the slice. Figure $2 \mathrm{~A}$ shows a typical neocortical coronal slice overlaid with the projection of a grid. The grid pattern in Figure 2A uses about 25\% of the DMD surface and is large enough to cover many neighboring columns and all six layers of the barrel cortex of a mouse.

A relatively high light intensity is required to photolytically release glutamate at concentrations sufficient to excite neurons. UV light power density, measured with an optical power meter (Thorlabs, Newton, NJ, USA) at the focal plane of the projection objective, was on average $250 \mathrm{~mW} / \mathrm{mm}^{2}$. This corresponds to a total absorption of $40 \%$ of the optical power in the projection light path, the majority of which is absorbed by the objective. Power was measured after 1-h of laser warm up before an experiment and immediately following an experiment and remained stable 


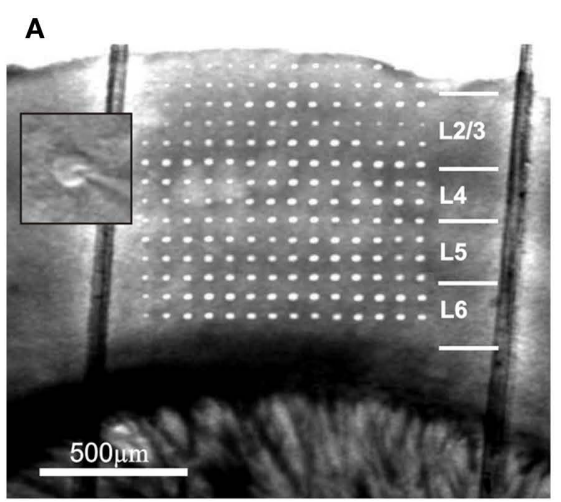

C

2.5mM MNI-glutamate

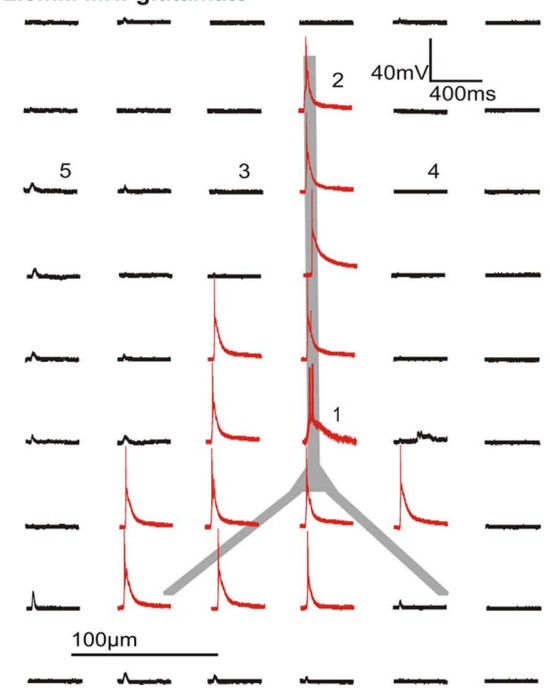

FIGURE 2 | Optical and physiological resolution of DLP uncaging. (A)

Overlay of a grid projection using approximately $25 \%$ of the DMD surface and a photomicrograph of a neocortical slice demonstrating the area of slice tissue that can be covered by DMD photostimulation. The two vertical lines are nylon strings used to hold the slice in place. The inset shows the center of the microscope's field of view at high magnification with a patched neuron. The position of the projected DMD image and the center of the field of view were fixed, allowing alignment of photostimulation with the patched cell. (B) Demonstration of 3-pixel $(8.1 \mu \mathrm{m})$ optical resolution of the DLP photostimulation system. The DMD-reflected UV light is projected onto a blue-painted coverslip bleaching the paint. The top panel shows 1-pixel wide bars, separated by gaps of the indicated width. The trace at the bottom plots the average numerical gray

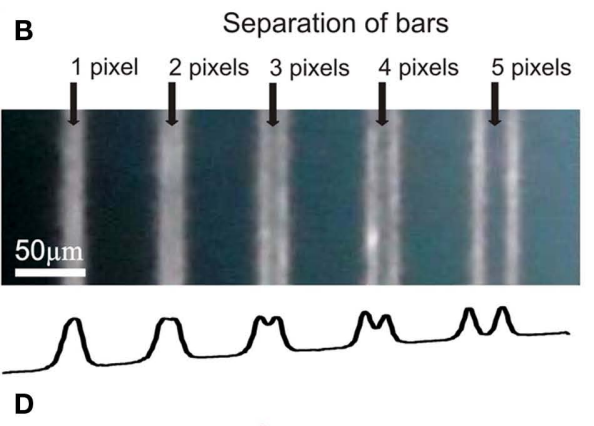

D

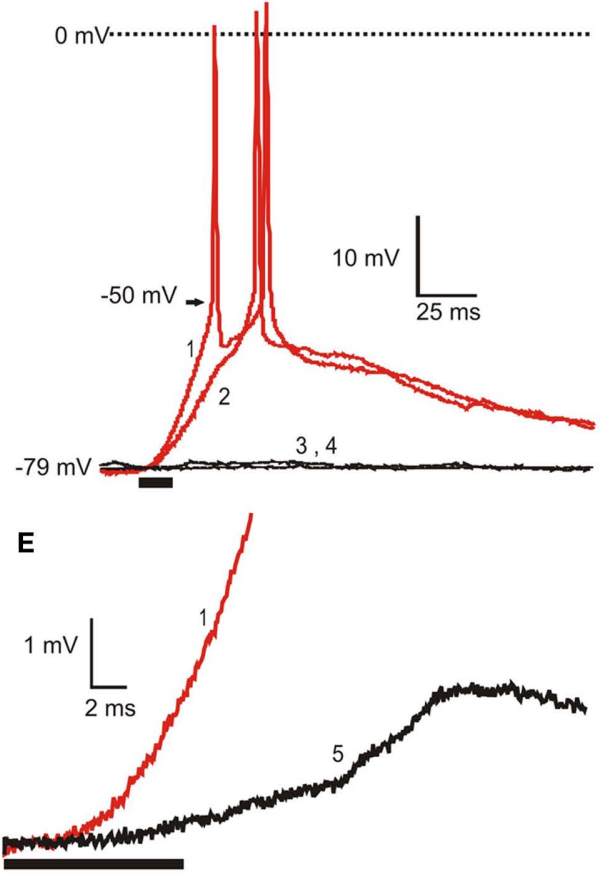

levels along the horizontal axis. (C) With $2.5 \mathrm{mM}$ MNI-glu in the bath solution, photostimuli of $8 \times 8$ pixels (micromirrors) induce action potentials in a layer $2 / 3$ pyramidal cell at sites aligned anatomically to the cell body or dendrites. The traces represent the membrane potential response elicited by photostimulation at each site. (D) Expanded views of the traces marked with numbers in (C), showing action potentials elicited by photostimulation of the distal dendrite (2) and soma (1) of a layer 2/3 pyramidal cell as well as the absence of both subthreshold and suprathreshold responses to photostimulation of adjacent sites $(3,4)$ on either side of the apical dendrite. The timing and duration of photostimulation $(10 \mathrm{~ms})$ is indicated with a black bar beneath the traces. (E) Synaptically induced responses (5) occur several milliseconds later than responses to direct stimulation (1).
( $<2 \%$ power change). The $\mathrm{x}-\mathrm{y}$ optical resolution of this system was demonstrated by projecting patterns onto a quartz coverslip painted with blue permanent marker placed painted side down in the perfusion chamber. The UV light bleached permanent imprints of the projected DMD image. Single-pixel-wide bars were distinguishable when separated by 3 pixels, suggesting a maximum optical resolution of $8.1 \mu \mathrm{m}$ (Figure 2B). Using the peak wavelength of the laser $(355 \mathrm{~nm})$ and the numerical aperture of the projection objective (0.25), The Rayleigh criterion calculates the theoretical resolution:

$\mathrm{R}=1 /(2 * \mathrm{NA})=335 \mathrm{~nm} /(2 * 0.25)=710 \mathrm{~nm}$.
When the bars are separated by 3 pixels, the minimum of the first diffraction order of one bar corresponds to the maximum of the first diffraction order of the neighboring bar. Thus, the empirically determined Rayleigh criterion is 3 pixels $(8.1 \mu \mathrm{m})$, which is larger than the calculated optical resolution.

The technical limits of photostimulation patterns in this system are set by the maximum number of frames in a sequence (1365), the number of pixels or mirrors on the DMD $(1024 \times 768)$, pixel size $\left(2.7 \mu \mathrm{m}^{2}\right)$, and the maximum frame rate (>13 kfps). Within these constraints, any sequence of 2-dimensional frames can be projected as UV photostimulation patterns. For any one sequence, the frames 
can be repeated in any order determined by the experimenter. This includes repetitions of the same frame as often as desired. The frame sequence can be played back with any frame rate and any interval between frames down to the maximum temporal resolution of the system (limited by the USB controller's speed).

\section{DETERMINING THE PHYSIOLOGICAL RESOLUTION OF DLP PHOTOSTIMULATION}

Ultraviolet light scatters significantly in living brain tissue and the bathing solution. Since scattered light also uncages glutamate, the actual physiological resolution of the photostimulation system is considerably lower than the maximal optical resolution measured with homogenous, thin projection media. The spatial resolution for the optical control of spiking activity was determined with $2.5 \mathrm{mM}$ caged 4-methoxy-7-nitroindolinyl-caged-L-glutamate (MNI-glu) in the bath solution. In order to maximize the probability of eliciting spike responses we used the highest concentration of caged glutamate reported in the literature so far. A concentration of $2.5 \mathrm{mM}$ is widely reported to be stable in solution with no detrimental effects to cells and is the only concentration we used in experiments (i.e., Losonczy et al., 2008). No noticeable changes in photostimulation efficacy or strength was apparent for the length of the experiment, given that each site was allowed to recover by not being stimulated more than once every $30 \mathrm{~s}$. This suggests a relatively stable concentration of caged glutamate in the bath for the length of the experiment.

The cell body and direction of the apical dendrite is easily seen with IR Hoffman-modulation contrast enhancement (Figure 2A inset). The position of the projected pixels relative to the center of the field of view of the microscope is fixed. The position of the cell relative to the DLP sites can thus be determined visually by centering the cell on a cross hair in the microscope's visual field. A grid of $21.6 \mu \mathrm{m} \times 21.6 \mu \mathrm{m}(8 \times 8$ pixels $)$ photostimulation sites with $86 \mu \mathrm{m}$ spacing (32-pixel) anatomically oriented over the soma or dendrites of layer $2 / 3$ (L2/3) cells reliably induced action potentials, while sites not aimed at the soma or dendrites did not (Figures 2C,D). With this spacing, neighboring stimulus sites had non-overlapping glutamate release ( $n=3$ cells; Figures 2 C,D) creating clearly distinct physiological responses (e.g., a postsynaptic response to stimulation at one site but not adjacent sites). This suggests an upper limit of the physiological resolution of $86 \mu \mathrm{m}$, which is comparable to most other UV uncaging systems designed to induce action potentials in presynaptic cells for circuit mapping purposes (Callaway and Katz, 1993; Dantzker and Callaway, 2000).

Using smaller spots $(6 \times 6,4 \times 4$ pixels, etc. $)$, action potentials induction was unreliable. Similarly, dwell times shorter than $10 \mathrm{~ms}$ would not reliably induce spike firing. A dwell time of $10 \mathrm{~ms}$ was therefore used in all subsequent experiments. The ability to reliably elicit action potentials at the highest possible spatial resolution was a major constraint and thus determined the optimal stimulus parameters used in all subsequent experiments.

In order to determine whether action potentials were elicited by direct stimulation of the recorded cell or via synaptic input from activated presynaptic cells we compared the time courses of postsynaptic potentials. Analysis of excitatory postsynaptic responses elicited by action potentials in presynaptic neurons (Figure 2E) shows that synaptically elicited excitatory postsynaptic potentials
(EPSPs) have post-stimulus onset times several milliseconds later than responses elicited by stimuli directly targeting the cell. The two response types were thus clearly distinct and action potentials were the result of direct photostimulation of the recorded cell. If subthreshold responses are of interest, as in many other uncaging applications, the physiological resolution will likely be higher due to the potential for using smaller groups of mirrors and shorter dwell times. However, the particular strength of the described method is that the system can be used to control spatiotemporal spiking activity. We thus focus on the parameters relevant for eliciting spike firing in the targeted neurons.

\section{PROPAGATION OF SYNCHRONOUS ACTIVITY FROM LAYER 4 TO LAYER 2/3}

Simulated neuronal networks have been extensively studied as a model for the propagation of neuronal signals (Diesmann et al., 1999; Van Rossum et al., 2002; Kumar et al., 2010). However, experimental evidence of neuronal signal propagation, especially of synchronous firing, is very limited due to technical constraints. A recent modeling study based on experimentally determined circuit parameters suggested that synchronous spike firing in L4 neurons may play an important role in driving L2/3 neurons (Sarid et al., 2007). However, direct experimental evidence of this finding has been technically unattainable. The following experiment was designed to investigate the role of synchrony for the propagation of activity from layer 4 of the whisker barrel cortex to layer $2 / 3$. Whole-cell patch-clamp recordings were obtained from layer $2 / 3$ pyramidal cells $(n=12)$ while photostimulating layer 4 neurons with varying degrees of synchrony.

Only cells with a resting membrane potential (RMP) more negative than $-55 \mathrm{mV}$ and with action potential peaks exceeding $0 \mathrm{mV}$ were included in the final analysis. Firing properties and recording rundown were monitored with a current-voltage response protocol (500 ms current steps incremented from $-200 \mathrm{pA}$ to $+400 \mathrm{pA}$ in $50 \mathrm{pA}$ increments with $2 \mathrm{~s}$ between sweeps). In all analyzed cells, $\mathrm{RMP}$ remained stable at an average $-83 \mathrm{mV} \pm 7 \mathrm{mV}$, and the initial spike peak reached $+7 \mathrm{mV} \pm 8 \mathrm{mV}$.

Six to 10 sites within L4 were selected for photostimulation (Figures 3A, 4B). The position of photostimulation sites was confirmed in video recordings filmed with a CCD camera (Hamamatsu C2400) through the microscope's $5 \times$ objective. Light flashes for each UV stimulus were clearly visible on a video monitor connected to the CCD camera. The UV laser emits light in the visible spectrum, albeit at low intensities, and the slices auto-fluoresce under UV illumination. Both components are likely to contribute to the visibility of the light stimuli in the CCD video system. Photostimulus onset times (signaled by a TTL-format DLP output) were digitized together with the membrane potential data. Activation of each individual L4 photostimulation site resulted in a measurable membrane potential responses starting $10-15 \mathrm{~ms}$ after the onset of photostimulation with the peak of the response occurring at $26.8 \pm 5.5 \mathrm{~ms}$, reflecting both the latency to induce action potentials in the presynaptic cell and the action potential conduction and synaptic release latencies.

In the example cell shown in Figure 3, close examination of the membrane potential traces revealed clusters of EPSP (Figures 3B,C). The peak amplitude of isolated EPSPs (from three 
A

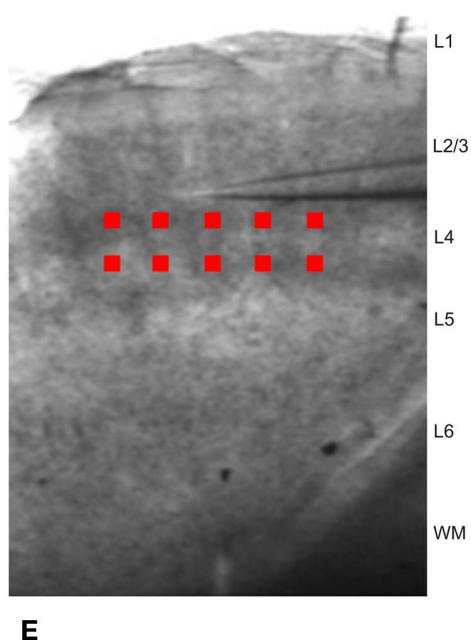

E

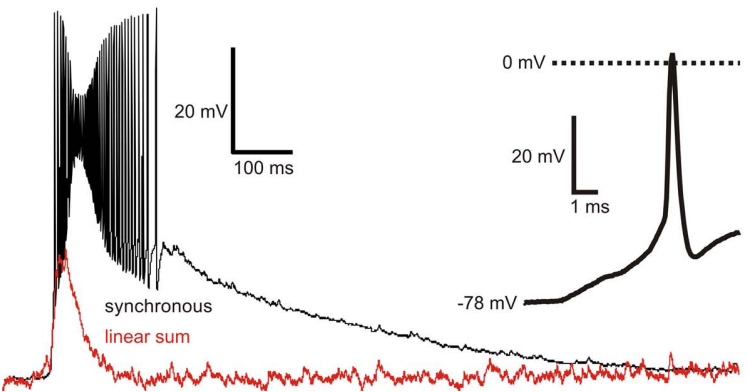

B

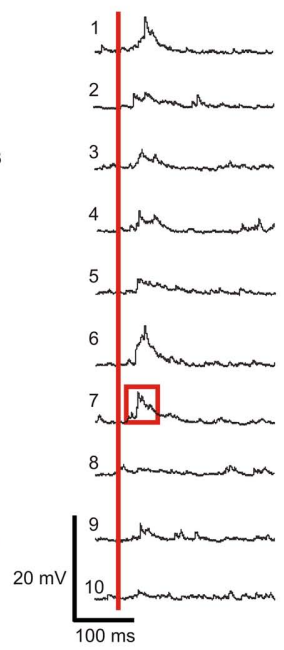

$+\cdots$
C

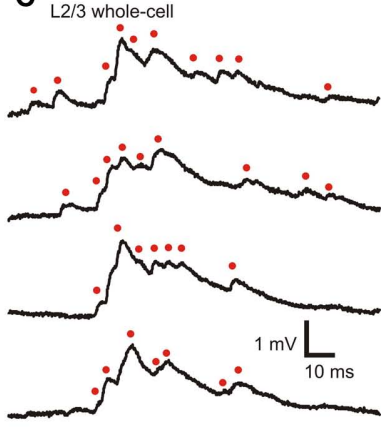

D

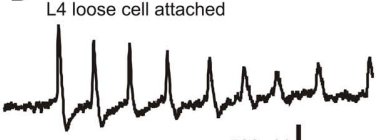

$500 \mu \mathrm{V} L$

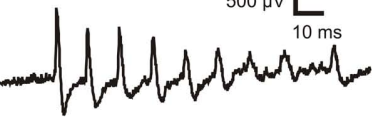

|

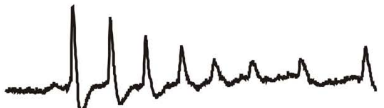

FIGURE 3 | Example of layer 2/3 pyramidal cell membrane potential responses to layer 4 photostimulation with 10 stimuli applied sequentially and synchronized. (A) Position and size of photostimulation sites (red squares) within $L 4$. (B) $L 2 / 3$ membrane potential responses to sequential activation of individual sites (30 s inter-stimulus interval, $10 \mathrm{~ms}$ dwell time) with the same scale as in (E). The onset of photostimulation is marked with a red vertical bar. (C) Expanded view of trace 7 in (B) (area outlined by red box) with three additional responses from subsequent trials shown in the three lower traces.
Presumed single or compound EPSPs are marked with red dots. (D) L4 spiking responses to direct somatic photostimulation showing that each stimulus typically elicits multiple action potentials. The time window relative to photostimulation is identical to that in (C,E) Supralinear L2/3 membrane potential responses to activation of all 10 sites with perfect synchrony. Red trace represents the linear sum of the individual responses shown in (B). Inset shows expanded view of the first spike in the burst of response spikes. Bath concentration of caged Glu was $2.5 \mathrm{mM}$. cells, 15 EPSPs each, $n=45$ ) was $0.66 \pm 0.32 \mathrm{mV}$, a $20-80 \%$ rise time of $1.01 \pm 0.66 \mathrm{~ms}$, a $80-20 \%$ decay time of $4.94 \pm 2.23 \mathrm{~ms}$, and an area under the curve of $3.2 \pm 0.9 \mathrm{~ms} * \mathrm{mV}$. These EPSP parameters have been previously determined in rat with paired recordings of layer $2 / 3$ pyramidal neurons and layer 4 spiny neurons (Feldmeyer et al., 2002) and are in close agreement with the result of singlesite photostimulation experiments. The advantage with this system over paired recording techniques is that a large number of presynaptic cells can be quickly and efficiently screened and the investigation of the temporal integration of multiple presynaptic cells is possible.

We also performed loose cell attached recordings of L4 cells $n=12$ ) using the same photostimulation pattern applied during $\mathrm{L} 2 / 3$ recordings. These recordings thus reveal the presynaptic activity driving the responses observed in L2/3 neurons. A typical response to photostimulation of this type is shown in Figure 3D. L4 cells responded to somatic photostimulation with a burst of spikes with a maximum instantaneous frequency of $(\sim 150 \mathrm{~Hz}$. Action potentials in L4 cells were previously found to reliably induce EPSPS in synaptically coupled L2/3 cells with a failure rate of ( $~ 5 \%$ in rat; Feldmeyer et al., 2002). The recordings by Feldmeyer et al. (2002) also revealed that a fast train of action potentials in a single synaptically coupled L4 cell results in a reliable constant-amplitude (or slightly adapting, depending on the frequency) train of EPSPs in the postsynaptic L2/3 cell (Feldmeyer et al., 2002). The response in Figure 3C, however, shows a tightly packed ensemble of EPSPs of varying amplitudes. This suggests that L4 photostimulation activates multiple presynaptic L4 cells within each site and that converging input from $\mathrm{L} 4$ onto $\mathrm{L} 2 / 3$ results in membrane responses like the one shown in Figure 3C.

To obtain a rough estimate of the number of synaptically coupled cells stimulated per stimulation site, we calculated the area under the compound EPSP (from the onset of photostimulation to $200 \mathrm{~ms}$ after the photostimulation) of the strongest $\mathrm{L} 2 / 3$ response ( $94.5 \mathrm{~ms} * \mathrm{mV}, A_{\text {response }}$ ) and divided by the product of the average area under the curve of the isolated EPSPs $\left(3.2 \mathrm{~ms} * \mathrm{mV}, A_{\text {epsp }}\right)$ and the number of EPSPs $\left(n_{\text {epsp }}\right)$ that can be expected when each 
presynaptic neuron fires a single action potential (maximum case) or each presynaptic neuron fires a $150-\mathrm{Hz}$ burst of action potentials (minimum case) $\left[A_{\text {response }} /\left(n_{\text {epsp }} * A_{\text {epsp }}\right)\right.$, see Table 1$]$.

These estimates are consistent with anatomical data. Based on neuronal density measurements in the mouse neocortex (Schuz and Palm, 1989) of $9.2 \times 10^{4}$ neurons per $\mathrm{mm}^{3}$, or a maximum of $2.5 \times 10^{5}$ neurons per $\mathrm{mm}^{3}$ if the higher density of neurons in L4 of granular cortex is considered (Tsai et al., 2011), there are 8.6-23.3 neurons underneath an $8 \times 8$-pixel $(21.6 \mu \mathrm{m} \times 21.6 \mu \mathrm{m})$ photostimulation site, assuming a $200-\mu \mathrm{m}$ thick core of healthy neurons in a 300- $\mu \mathrm{m}$ thick slice. Due to light scatter and glutamate diffusion the physiologically effective size of a photostimulation site is closer to a cylinder with an estimated $43 \mu \mathrm{m}$ radius (Figure 2), leading to an estimated maximal number of 106.9-290.5 neurons per photostimulation site. The probability of synaptic connections between L4 and L2/3 neurons in acute slices is estimated to be 28\% (connection ratio 1:3.6 in rat; Bannister and Thomson, 2007), resulting in 2.4-81.3 presynaptic L4 neurons per photostimulation site.

It may be possible to reduce the number of neurons activated at each site with techniques analogous to minimal photostimulation (Bureau et al., 2008) by decreasing the dwell time of each photostimulation site or by adjusting the grayscale modulation of the mirrors in each site. Allowing for reasonable recovery times between trials (20 s or more) these steps are prohibitively timeconsuming for patch-clamp experiments with 10 sites but could be performed using sharp electrodes or perforated patch techniques. For example, if on average three trials of five dwell time settings are required to determine the minimal photostimulation settings for 10 sites, the time required is:

10 sites $\times 5$ settings $\times 3$ trials $\times 20 \mathrm{~s}=50 \mathrm{~min}$.

A typical whole-cell recording will be good for between 30 and $60 \mathrm{~min}$, leaving little or no time for an experiment after stimulus parameters are determined. Sharp or extracellular recordings can last a little longer, but have their own disadvantages and were not performed here.

After the sequential scan of photostimulation sites, the degree of synchrony in layer 4 was gradually increased by shortening the inter-stimulus interval. In most of the L $2 / 3$ cells ( $n=10$ out of 12 ), temporal summation of L4 synaptic input resulted in suprathreshold depolarizations for highly synchronous L4 activity (intervals between individual stimuli: 0 and $2 \mathrm{~ms}$, synchrony: $\Delta t=0$ and $14 \mathrm{~ms}$, Figure 4A) and subthreshold depolarizations for less synchronized L4 activity (inter-stimulus intervals of $5 \mathrm{~ms}$ or longer or synchrony of $\Delta t \geq 35 \mathrm{~ms}$, Figure $4 \mathrm{~A}$ ). This finding strongly suggests that a sub-population of L2/3 neurons is highly sensitive to temporal precision of synchronized activity in layer 4 .

Table 1 | Maximum and minimum estimates of the number of presynaptic neurons stimulated in each $\mathrm{L} 4$ site.

\begin{tabular}{lllll}
\hline Case & $\boldsymbol{n}_{\mathrm{AP}}$ & EPSP failure rate & $\boldsymbol{n}_{\text {epsp }}$ & $\boldsymbol{n}_{\text {pre }}$ \\
\hline Minimum & $30(150 \mathrm{~Hz} * 200 \mathrm{~ms})$ & $0 \%$ & 30 & 0.98 \\
Maximum & 1 & $10 \%$ & 0.9 & 32.81
\end{tabular}

$n_{A P^{\prime}}$ action potentials per presynaptic neuron; $n_{\text {epsp' }}$ excitatory postsynaptic potentials per presynaptic neuron; $n_{\text {pre' }}$ analytical estimate of the number of presynaptic neurons.

\section{DISCUSSION}

The use of light for the manipulation of neuronal activity, either through photolytic release of neurotransmitters or activation of light-sensitive ion channels, has opened up new vistas in experimental neuroscience. Recent years have seen rapid progress in the development of new caged compounds and light-sensitive ion channels, providing unprecedented new opportunities for the experimental investigation of brain function. To take full advantage of these new experimental tools, we have developed a light stimulation technology that allows emulation of the complexity of in vivo spatiotemporal neuronal activity patterns under in vitro experimental conditions. The main advantage of the DLP-based stimulation method described here is that it allows massively parallel stimulation compared to systems using fast moving single beams that are limited to sequential stimulation (Boucsein et al., 2005).

Likely the most common alternative technique for manipulating synaptic inputs, electrical stimulation with multiple electrodes, has severe disadvantages over parallel light stimulation. One is the much lower number of possible stimulation sites. With multi-electrode
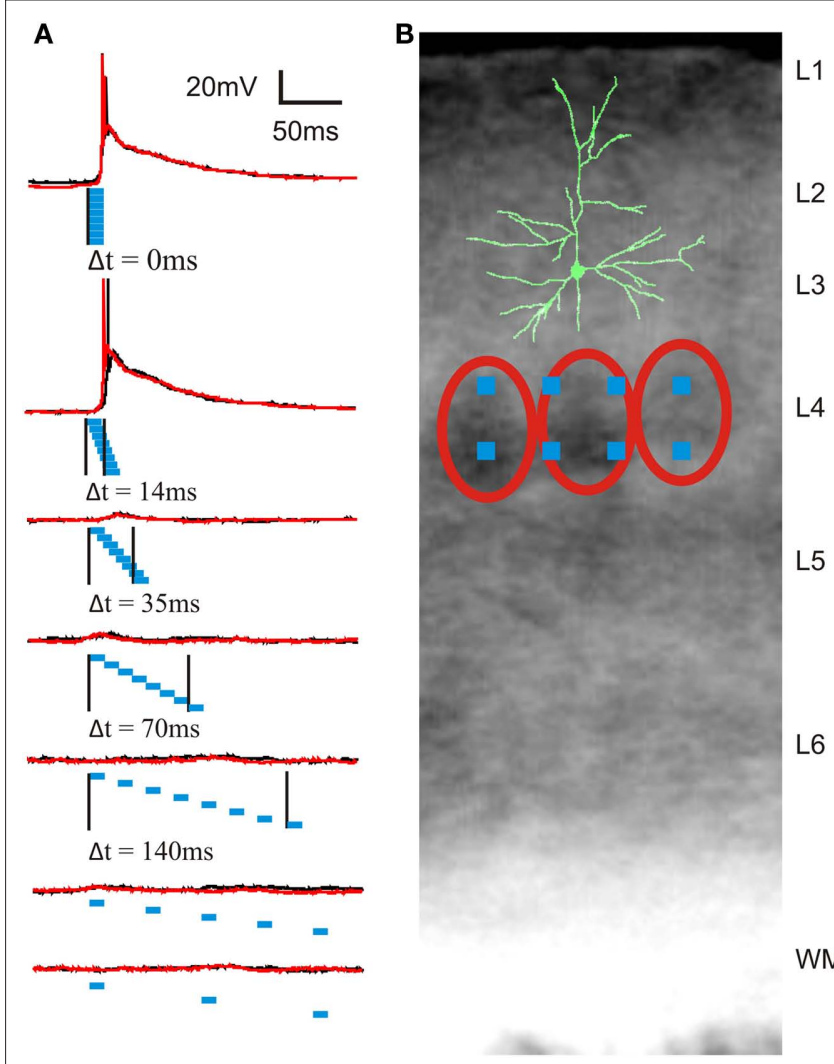

FIGURE 4 | Example of layer 2/3 pyramidal cell membrane potential responses to photostimulation of eight sites in layer $\mathbf{4}$ with different degrees of synchrony. (A) Membrane potential responses (two trials shown as red and black traces) to photostimulation with seven different degrees of synchrony. Blue rectangles represent timing and duration of eight photostimuli. Black vertical lines mark the times of onset of the first and last photostimulus, corresponding to the time window or temporal precision of synchronization ( $\Delta t=0$ to $350 \mathrm{~ms}$ ). Duration of each individual photostimulus was $10 \mathrm{~ms}$. (B) Location of photostimulation sites in a coronal barrel cortex slice with overlay of a partially reconstructed biocytin-filled cell. Red ovals outline cell-dense barrels. 
arrays, the typical number of sites is less than or around 100 electrodes, compared to $>780,000$ in the parallel photostimulation system described here. Furthermore, there is no flexibility in the spatial arrangement or the size of stimulation sites, as electrodes are arranged on a fixed grid and the location of sites on the slice is determined by the placement of the slice on the grid. Another disadvantage of electrical stimulation is its poor anatomical specificity as it mostly activates fibers of passage and not somata and dendrites (Nowak and Bullier, 1998a,b) and results also antidromic activation of neurons. This is particularly problematic in the neocortex, where a high density of axons originating from multiple brain regions passes through every cortical layer. Thus, parallel photostimulation provides several qualitative and quantitative advantages over electrical stimulation techniques.

The parallel photostimulation system described here allows precise spatiotemporal manipulation of action potential firing and subthreshold neuronal activity at a broad range of anatomical scales. Because the system's $>780,000$ light beams are independently controlled, the illumination duration for each beam or stimulation site can be tuned to produce subthreshold responses or action potential firing, independent from the number of stimulation sites and the stimulation frequency. This is a major advantage over single-beam systems, where illumination duration, the number of sites and the stimulation frequency variables are interdependent.

Because the system is based on computer-controlled DLP technology, it allows for convenient programming of photostimulation patterns using GUIs. Stimulation sites can be selected visually or preprogrammed patterns can be positioned to target specific cortical layers or the area of an individual dendrite. These unique features of parallel light stimulation make this technique well suited for the investigation of the complex spatiotemporal neuronal activity patterns and their functional significance for dendritic integration, synaptic plasticity, and the propagation of activity through the neuronal network. A unique strength of the new system is that the degree of synchrony as well as the size and spatial distribution of neuronal populations can be easily manipulated. The system is thus ideally suited to address long-standing questions about the propagation of oscillatory and synchronous activity in a biological network of randomly firing neurons (Diesmann et al., 1999). Experiments based mostly on intracellular current injections strongly suggest that synchronous synaptic input is coupled to precisely timed action potential initiation in both cortical (Nettleton and Spain, 2000; Rodriguez et al., 2007) and hippocampal (Gasparini et al., 2004) pyramidal neurons. By stimulating in layer 4 and recording intracellular responses in layer $2 / 3$, we demonstrated the use of DLP photostimulation to investigate how synchronous activity in one layer of cortex influences spiking activity in another layer. The membrane potentials of cortical neurons in vivo exhibit rapid voltage fluctuations caused by ongoing activity of thousands of synaptic inputs (Stern et al., 1997; Steriade et al., 2001). This background activity influences the physiological properties and action potential output of neurons (Chance et al., 2002; Fellous et al., 2003; Léger et al., 2005). With parallel light stimulation it is possible to independently control random background activity and synchronous activity through separate groups of stimulation sites. This provides new approaches for the investigation of the influence of ongoing background activity on the generation and propagation of synchronous spiking activity.
Within the whisker barrel cortex, projections from L4 to L2/3 were shown to be one of the strongest interlaminar pathways (Hooks et al., 2011) with L2/3 neurons receiving converging inputs from approximately 300-400 spiny L4 neurons (Lubke et al., 2003). Modeling studies based on experimentally determined synaptic parameters (Feldmeyer et al., 2002) and the known in vivo firing probability of L4 neurons after whisker deflection (Brecht and Sakmann, 2002) suggest that the peak membrane potential deflection resulting from the compound EPSPs is not sufficient to drive L2/3 pyramidal neurons to fire spikes. However, L2/3 pyramidal cell spike firing in response to whisker stimulation is well documented (e.g., Simons, 1978). The authors of the modeling study acknowledge this discrepancy and suggest several possible solutions, one of which is the existence of spiking synchrony in L4 which would drive L2/3 neurons with greater efficiency (Sarid et al., 2007) as is the case with synchronously firing thalamic neurons driving L4 (Bruno and Sakmann, 2006). Our experiments support this hypothesis by showing that synchronous activation of L4 neurons preferentially caused L2/3 pyramidal neurons to fire action potentials.

Digital light processing-photostimulation of light-sensitive ion channels to manipulate the activity of genetically defined subsets of neurons has been used to investigate odorant coding in the olfactory bulb in vivo (Dhawale et al., 2010) and in cultured neurons with simultaneous optical imaging of calcium activity (Wang et al., 2007). The introduction of multiple stimulation wavelengths is technically straightforward and will allow the independent control of distinct groups of neurons expressing different light-sensitive ion channels gated by different wavelengths. While light-sensitive ion channel assisted circuit mapping has many advantages when compared to neurotransmitter uncaging, there are several disadvantages. First, activation of these channels has slower kinetics than glutamatergic activation, so temporal precision of action potential induction is somewhat limited. As an example, inactivation of channelrhodopsin-2 (CHR2) is about 10 times slower than its activation, resulting in plateau depolarizations and multiple spikes in response to a single light pulse. Also, CHR2-driven spike trains cannot exceed $40 \mathrm{~Hz}$, therefore, precise control of neural activity into the gamma band of neural activity is not possible (Ishizuka et al., 2006; Gunaydin et al., 2010). Recently, multiple reengineered variant of CHR2 with improved kinetics have started to overcome this obstacle (Lin et al., 2009; Gunaydin et al., 2010), although genetic constructs or a transgenic mouse line with these kinetics are not yet readily available.

Another disadvantage is due the subcellular distribution of ectopic light-sensitive ion channels in transgenic animals and most cell culture preparations. CHR2 is indiscriminately and uniformly distributed in the plasma membrane, while glutamate receptors are limited to the dendrites and cell bodies of neurons. Blue light activation of CHR2, for example, activates both cell bodies and axons of passage, making it impossible to precisely determine where the synaptic connection originates. This is also true of cut axon terminals in slice preparations, which may still be activated and release neurotransmitter, giving potentially confounding results in attempts to elucidate microcircuitry. This has been partially overcome by adding a myosin-dependent targeting sequence to the transgene, restricting CHR2 expression to dendrites (Lewis et al., 2009) although again, a transgenic mouse or construct with this modification is not yet readily available and requires multiple 
additional complex steps to express with retroviral constructs. In light of these limitations, it is more appropriate to classify the roles of intrinsically light-sensitive ion channels and uncaging-based photostimulation systems as complementary rather than competing technologies. In fact, these tools have been combined for optical induction of plasticity at single synapses (Zhang et al., 2008).

Recently developed holographic photostimulation techniques share some advantages with parallel photostimulation (Golan et al., 2009; Dal Maschio et al., 2010). However, they depend on a relatively slow light modulation technique (spatial light modulators) limiting the temporal precision to between 33 and $15 \mathrm{~ms}$. Current holographic systems are thus not capable of reproducing fast dynamic neuronal activity patterns. Their strength lies in the investigation of spatial integration through the ability to define uncaging locations in 3-dimensions. Another recently described UV DLP photostimulation system has been used for investigating dendritic integration of subthreshold stimuli (Liang et al., 2011). However, with the limited subcellular resolution of ultraviolet uncaging, UV photostimulation mostly activates extrasynaptic receptors, which may have implications in dendritic processing. For this reason, it is the author's belief that this technology is more strongly suited for presynaptic photostimulation of large neuronal networks, which allows for the investigation of spatiotemporal synaptic integration in the context of intact neuronal microcircuits.

The massively parallel optical manipulation of spiking activity in anatomically distributed subsets of neurons is thus a unique capability and strong tool for the investigation of information processing and coding performed by large neuronal networks. DLPbased photostimulation opens up important new experimental opportunities to investigate the complex spatiotemporal neuronal interactions underlying the dynamic activity patterns that are characteristic of the conscious brain.

\section{ACKNOWLEDGMENTS}

We would like to thank Michael Nguyen for excellent technical advice and manufacturing of custom parts and L. Wang for his generous help with staining procedures. This research was supported by NIH grant R21MH079315-01A1 (to Detlef H. Heck, William J. Spain, and Robert C. Foehring), by funding from the University of Tennessee Health Science Center Neuroscience Institute and a VA Merit Review (to William J. Spain). The content of this publication is solely the responsibility of the authors and do not necessarily represent the official views of the $\mathrm{NIH}$.

\section{REFERENCES}

Bannister, A. P., and Thomson, A. M. (2007). Dynamic properties of excitatory synaptic connections involving layer 4 pyramidal cells in adult rat and cat neocortex. Cereb. Cortex 17, 2190-2203.

Boucsein, C., Nawrot, M., Rotter, S., Aertsen, A., and Heck, D. (2005). Controlling synaptic input patterns in vitro by dynamic photo stimulation. J. Neurophysiol. 94, 2948-2958.

Brecht, M., and Sakmann, B. (2002). Dynamic representation of whisker deflection by synaptic potentials in spiny stellate and pyramidal cells in the barrels and septa of layer 4 rat somatosensory cortex. J. Physiol. 543, 49-70.

Bruno, R. M., and Sakmann, B. (2006). Cortex is driven by weak but synchronously active thalamocortical synapses. Science 312, 1622-1627.

Bureau, I., Shepherd, G. M., and Svoboda, K. (2008). Circuit and plasticity defects in the developing somatosensory cortex of FMR1 knock-out mice. J. Neurosci. 28, 5178-5188.

Callaway, E. M., and Katz, L. C. (1993). Photostimulation using caged glutamate reveals functional circuitry in living brain slices. Proc. Natl. Acad. Sci. U.S.A. 90, 7661-7665.

Chance, F. S., Abbott, L. F., and Reyes, A. D. (2002). Gain modulation from background synaptic input. Neuron 35, 773-782.

Dal Maschio, M., Difato, F., Beltramo, R., Blau, A., Benfenati, F., and Fellin, T. (2010). Simultaneous two-photon imaging and photo-stimulation with structured light illumination. Opt. Express 18, 18720-18731.

Dantzker, J.L., and Callaway, E. M. (2000). Laminar sources of synaptic input to cortical inhibitory interneurons and pyramidal neurons. Nat. Neurosci. 3, 701-707.

Dhawale, A. K., Hagiwara, A., Bhalla, U. S., Murthy, V. N., and Albeanu, D. F. (2010). Non-redundant odor coding by sister mitral cells revealed by light addressable glomeruli in the mouse. Nat. Neurosci. 13, 1404-1412.

Diesmann, M., Gewaltig, M. O., and Aertsen, A. (1999). Stable propagation of synchronous spiking in cortical neural networks. Nature 402, 529-533.

Feldmeyer, D., Lubke, J., Silver, R. A., and Sakmann, B. (2002). Synaptic connections between layer 4 spiny neurone-layer $2 / 3$ pyramidal cell pairs in juvenile rat barrel cortex: physiology and anatomy of interlaminar signalling within a cortical column. J. Physiol. 538, 803-822.

Fellous, J. M., Rudolph, M., Destexhe, A., and Sejnowski, T. J. (2003). Synaptic background noise controls the input/ output characteristics of single cells in an in vitro model of in vivo activity. Neuroscience 122, 811-829.

Gasparini, S., Migliore, M., and Magee, J. C. (2004). On the initiation and propagation of dendritic spikes in CA1 pyramidal neurons. J. Neurosci. 24, 11046-11056.

Georgopoulos, A. P., Kalaska, J. F., Crutcher, M. D., Caminiti, R., and
Massey, J. T. (1984). "The representation of movement direction in the motor cortex: single cell and population studies," in Dynamic Aspects of Neocortical Function, eds G. M. Edelman, W.M. Cowan, and W.E. Gall (New York: Wiley), 501-524.

Golan, L., Reutsky, I., Farah, N., and Shoham, S. (2009). Design and characteristics of holographic neural photo-stimulation systems. J. Neural Eng. 6, 066004.

Gunaydin, L. A., Yizhar, O., Berndt, A., Sohal, V. S., Deisseroth, K., and Hegemann, P. (2010). Ultrafast optogenetic control. Nat. Neurosci. 13, 387-392.

Gutierrez, R., Simon, S. A., and Nicolelis, M. A. (2010). Lickinginduced synchrony in the taste-reward circuit improves cue discrimination during learning. J. Neurosci. 30 , 287-303.

Hooks, B. M., Hires, S. A., Zhang, Y. X. Huber, D., Petreanu, L., Svoboda, K., and Shepherd, G. M. (2011). Laminar analysis of excitatory local circuits in vibrissal motor and sensory cortical areas. PLoS Biol. 9, e1000572. doi: 10.1371/journal.pbio. 1000572

Hubel, D. H., and Wiesel, T. N. (1968) Receptive fields and functional architecture of monkey striate cortex. J. Physiol. 195, 215-243.

Huetz, C., Philibert, B., and Edeline, J. M. (2009). A spike-timing code for discriminating conspecific vocalizations in the thalamocortical system of anesthetized and awake guinea pigs. $J$. Neurosci. 29, 334-350.
Ishizuka, T., Kakuda, M., Araki, R., and Yawo, H. (2006). Kinetic evaluation of photosensitivity in genetically engineered neurons expressing green algae light-gated channels. Neurosci. Res. 54, 85-94.

Kreiter, A. K., and Singer, W. (1996). Stimulus-dependent synchronization of neuronal responses in the visual cortex of the awake macaque monkey. J. Neurosci. 16, 2381-2396.

Kumar, A., Rotter, S., and Aertsen, A. (2010). Spiking activity propagation in neuronal networks: reconciling different perspectives on neural coding. Nat. Rev. Neurosci. 11, 615-627.

Léger, J. F., Stern, E. A., Aertsen, A., and Heck, D. H. (2005). Synaptic integration in the cortex shaped by network activity. J. Neurophysiol. 93, 281-293.

Lewis, T. L. Jr., Mao, T., Svoboda, K. and Arnold, D. B. (2009). Myosindependent targeting of transmembrane proteins to neuronal dendrites. Nat. Neurosci. 12, 568-576.

Liang, C. W., Mohammadi, M., Santos, M D., and Tang, C. M. (2011). Patterned photostimulation with digital micromirror devices to investigate dendritic integration across branch points. J. Vis. Exp. 49.

Lin, J. Y., Lin, M. Z., Steinbach, P., and Tsien, R.Y. (2009). Characterization of engineered channelrhodopsin variants with improved properties and kinetics. Biophys. J. 96, 1803-1814.

Losonczy, A., Makara, J. K., and Magee, J. C. (2008). Compartmentalized dendritic plasticity and input feature storage in neurons. Nature 452, 436-441. 
Lubke, J., Roth, A., Feldmeyer, D., and Sakmann, B. (2003). Morphometric analysis of the columnar innervation domain of neurons connecting layer 4 and layer $2 / 3$ of juvenile rat barrel cortex. Cereb. Cortex 13, 1051-1063.

Nettleton, J. S., and Spain, W. J. (2000). Linear to supralinear summation of ampa-mediated epsps in neocortical pyramidal neurons. J. Neurophysiol. 83, 3310-3322.

Nowak, L. G., and Bullier, J. (1998a). Axons, but not cell bodies, are activated by electrical stimulation in cortical gray matter I. Evidence from chronaxie measurements. Exp. Brain Res. 118, 477-488.

Nowak, L. G., and Bullier, J. (1998b). Axons, but not cell bodies, are activated by electrical stimulation in cortical gray matter II. Evidence from selective inactivation of cell bodies and axon initial segments. Exp. Brain Res. 118, 489-500.

Prut, Y., Vaadia, E., Bergman, H., Haalman, I., Slovin, H., and Abeles, M. (1998). Spatiotemporal structure of cortical activity: properties and behavioral relevance. J. Neurophysiol. 79, 2857-2874.

Riehle, A., Grån, S., Diesmann, M., and Aertsen,A. (1997). Spike synchronization and rate modulation differentially involved in motor cortical function. Science 278, 1950-1953.
Rodriguez, M. A., Aertsen, A., and Heck, D. H. (2007). Spike firing in cortical pyramidal neurons: effects of EPSC kinetics, input synchronization and background noise on spike timing. PLoS ONE 2, e319. doi: 10.1371/journal.pone.0000319

Salinas, E., and Sejnowski, T. J. (2001). Correlated neuronal activity and the flow of neural information. Nat. Rev. Neurosci. 2, 539-550.

Sarid, L., Bruno, R., Sakmann, B., Segev, I., and Feldmeyer, D. (2007). Modeling a layer 4-to-layer 2/3 module of a single column in rat neocortex: interweaving in vitro and in vivo experimental observations. Proc. Natl. Acad. Sci. U.S.A. 104, 16353-16358.

Schuz, A., and Palm, G. (1989). Density of neurons and synapses in the cerebral cortex of the mouse. J. Comp. Neurol. 286, 442-455.

Simons, D. J. (1978). Response properties of vibrissa units in rat SI somatosensory neocortex. J. Neurophysiol. 41,798-820.

Steinmetz, P. N., Roy, A., Fitzgerald, P. J., Hsiao, S. S., Johnson, K. O., and Niebur, E. (2000). Attention modulates synchronized neuronal firing in primate somatosensory cortex. Nature 404, 187-190.

Steriade, M., Timofeev, I., and Grenier, F. (2001). Natural waking and sleep states: a view from inside neocor- tical neurons. J. Neurophysiol. 85 , 1969-1985.

Stern, E. A., Kincaid, A. E., and Wilson, C. J. (1997). Spontaneous subthreshold membrane potential fluctuations and action potential variability of rat corticostriatal and striatal neurons in vivo. J. Neurophysiol. 77, 1697-1715.

Tsai, P. S., Kaufhold, J. P., Blinder, P., Friedman, B., Drew, P. J., Karten, H. J., Lyden, P. D., and Kleinfeld, D. (2011). Correlations of neuronal and microvascular densities in murine cortex revealed by direct counting and colocalization of nuclei and vessels. J. Neurosci. 29, 14553-14570.

Vaadia, E., Haalman, I., Abeles, M., Bergman, H., Prut, Y., Slovin, H., and Aertsen, A. (1995). Dynamics of neuronal interactions in monkey cortex in relation to behavioural events. Nature 373, 515-518.

Van Rossum, M. C., Turrigiano, G. G., and Nelson, S. B. (2002). Fast propagation of firing rates through layered networks of noisy neurons. J. Neurosci. 22, 1956-1966.

Wang, S., Szobota, S., Wang, Y., Volgraf, M., Liu, Z., Sun, C., Trauner, D., Isacoff, E. Y., and Zhang, X. (2007). All optical interface for parallel, remote, and spatiotemporal control of neuronal activity. Nano Lett. 7, 3859-3863.
Zhang, Y.P., Holbro, N., and Oertner, T. G. (2008). Optical induction of plasticity at single synapses reveals input-specific accumulation of alphaCaMKII. Proc. Natl. Acad. Sci. U.S.A. 105, 12039-12044.

Conflict of Interest Statement: Detlef H. Heck declares a competing financial interest: He holds a European patent on "Dynamic Simultaneous Multisite Photostimulation (DSMP)" (Nr. 05.019878.7). Jason Jerome, William J. Spain, Robert C. Foehring, and William E. Armstrong declare no competing financial interests.

Received: 07 May 2011; accepted: 08 August 2011; published online: 25 August 2011.

Citation: Jerome J, Foehring RC, Armstrong WE, Spain WJ and HeckDH (2011) Parallel optical control of spatiotemporal neuronal spike activity using high-speed digital light processing. Front. Syst. Neurosci. 5:70. doi: 10.3389/fnsys.2011.00070

Copyright (c) 2011 Jerome, Foehring, Armstrong, Spain and Heck. This is an open-access article subject to a nonexclusive license between the authors and Frontiers Media SA, which permits use, distribution and reproduction in other forums, provided the original authors and source are credited and other Frontiers conditions are complied with. 


\section{APPENDIX \\ CONSTANT VARIABLE DECLARATIONS NEEDED FOR NORMAL OPERATION OF IMPORTED DLL FUNCTIONS}

This document provides portions of the code critical for operating the DMD with a USB connection. This code requires that the dll provided with the ALP-3 kit is properly linked in visual studio (or any other development environment). Some of the code may require installation of .NET 3.5 .

\section{CONSTANT VARIABLE DECLARATIONS NEEDED FOR NORMAL OPERATION OF IMPORTED DLL FUNCTIONS}

//The C++ sample code provided with the ALP-3 documentation has many precompiler directives //(\#define) that give meaningful names to frequently used constants, such as return codes and //parameters for functions.

//

//In C\#, these constants must be declared as const ints to work. Below are a few examples. The //ALP-3 high-speed documentation provides a complete list of constants.

$/ / \mathrm{C}++$ code

/ /return codes

\#define ALP_OK 0x00000000

//no errors during execution

\#define ALP_PARM_INVALID 1005

//invalid parameter/argument

$\cdots$

//parameters/arguments for AlpDevInquire function

\#define ALP_DEVICE_NUMBER 2000 //return device serial number

\#define ALP_AVAIL_MEMORY 2003 / / return available RAM space

...

\section{/ / C\# code}

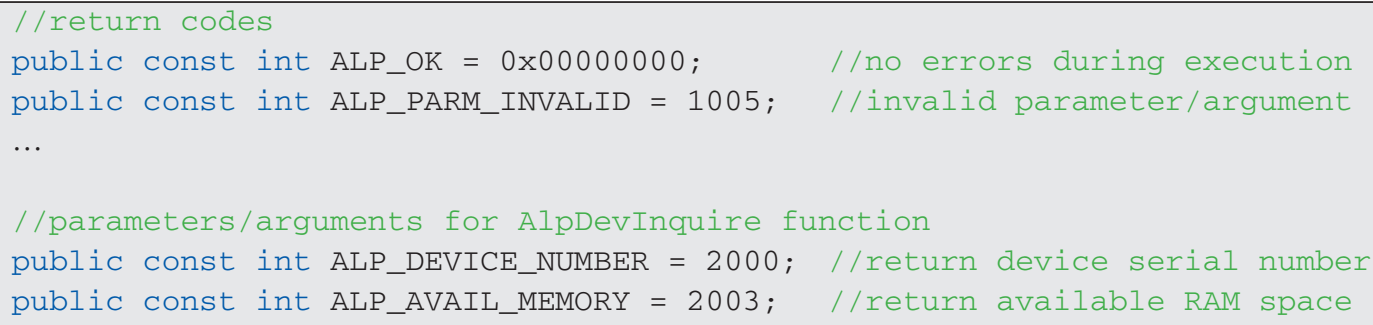

\section{EXAMPLE IMPORTATION AND MARSHALL OF ALP DLL FUNCTIONS INTO C\#}

//importing and marshalling of ALP functions requires correct C\# equivalents of C++ data types

//the following commonly used functions, as written below, import correctly.

[D1limport ("alp3.d11")]

public static extern int AlpDevHalt(IntPtr DeviceId);

[D1limport ("alp3.dll")]

public static extern int AlpDevFree(IntPtr DeviceId);

[D1limport ( "alp3.d11")] public static extern int AlpDevAlloc(int DeviceNum, int InitFlag, out IntPtr

DeviceIdPtr);

[D1limport ( "alp3.dll")] public static extern int AlpDevInquire(IntPtr DeviceId, int InquireType, out IntPtr UserVarPtr);

[D1limport ("alp3.dl1")]

public static extern int AlpSeqAlloc(IntPtr DeviceId, int BitPlanes, int PicNum, out IntPtr SequenceIdPtr);

[D1limport ( "alp3.dl1")]

public static extern int AlpseqTiming(IntPtr DeviceId, IntPtr SequenceID, int 
IlluminateTime, int PictureTime, int TriggerDelay, int TriggerPulsewidth, int VaDelay); [Dllimport ("alp3.dll")] public static extern int AlpSeqInquire(IntPtr DeviceId, IntPtr SequenceId, int InquireType, out IntPtr UserVarPtr);

[Dllimport ( "alp3.d11")] public static extern int AlpseqPut(IntPtr DeviceId, IntPtr SequenceId, int Picoffset, int PicLoad, byte $[$, , ] UserArrayPtr);

[Dllimport ( "alp3.dll")] public static extern int Alpseqfree(IntPtr DeviceId, IntPtr SequenceId);

\section{EXAMPLE INITIALIZATION OF DLP/ALP SYSTEM}

//The function below (initialize()) initializes USB communication with the DLP system and returns //information on the device state

public static void initialize()

\{

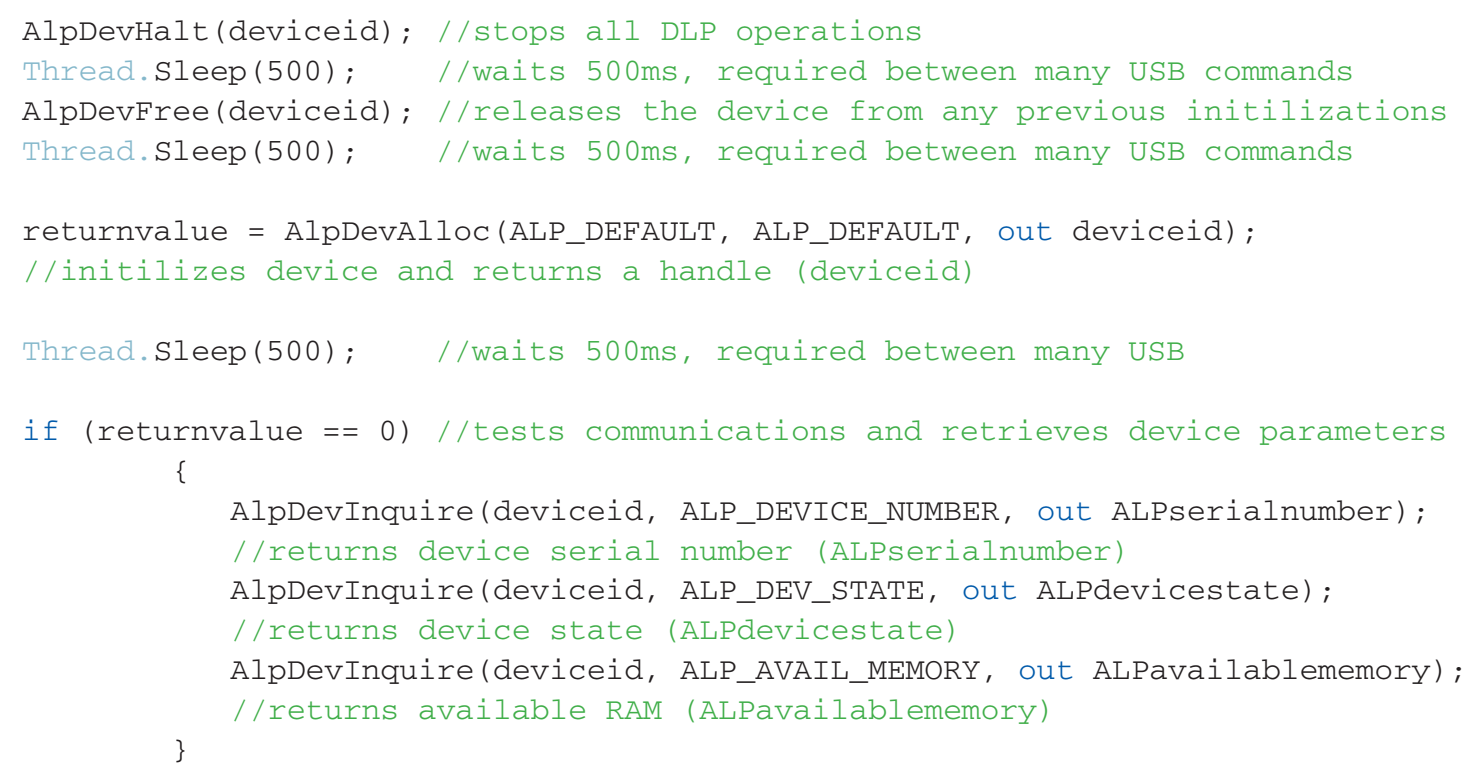

\section{EXAMPLE OF LOADING A SEQUENCE TO ALP RAM}

//allocates RAM on USB ALP board for loading a sequence with a specified number of frames $/ /$ (numberofstimuli) and returns a handle for the sequence (sequenceid) AlpSeqAlloc (deviceid, 1, numberofstimuli, out sequenceid);

//waits $500 \mathrm{~ms}$, required between many USB commands

Thread.Sleep (500);

//loads a sequence

for (int $i=0 ; i<$ numberofstimuli; $i++$ )

\{

//creates an array in the correct format for loading to ALP RAM

//bitmapheight and bitmap width correspond to the size of the micromirror array byte [, , loadedarray = new byte [1, bitmapheight, bitmapwidth] ;

//creates a bitmap and graphics object for programing sequences

//here, a the black bru sh creates an square of "on" mirrors

//with the coordinates and size specified with xstart, ystart, and patternsize 


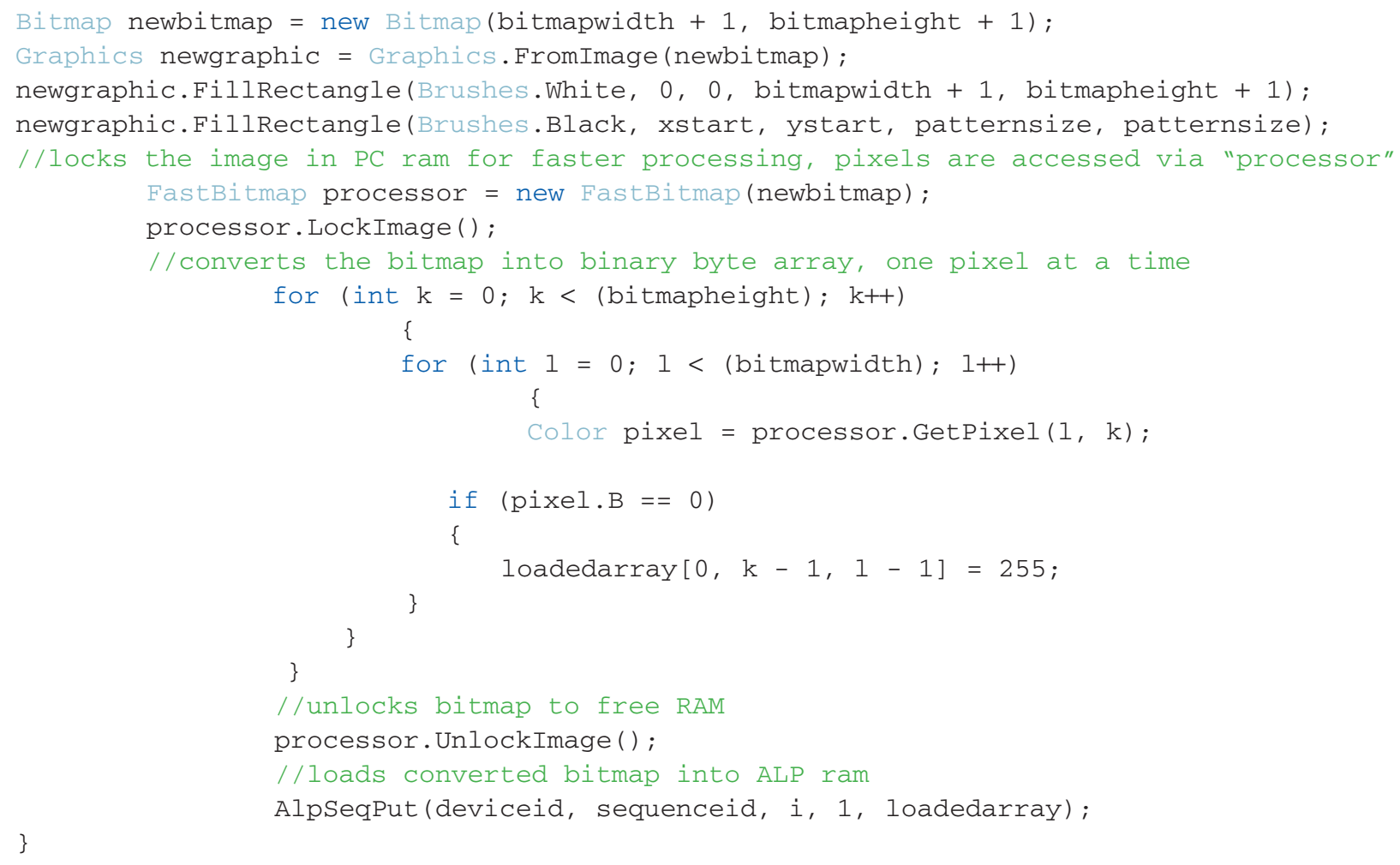

\section{EXAMPLE STARTING/STOPPING OF SEQUENCES WITH BUTTON CLICKS}

//event handler for click "startbutton,"

//this code requires the creation of a button object in Visual Studio, with the "click" action //linked to this eventhandler. The start button starts the specified sequence (sequenceid.

private void startbutton_Click(object sender, EventArgs e)

\{

AlpProjStart (deviceid, sequenceid);

\}

//event handler for click "stopbutton"

//this code requires the creation of a button object in Visual studio, with the "click" action //linked to this eventhandler. The stop button stops all activity on the specified device

$/ /($ deviceid).

private void stopbutton_Click(object sender, EventArgs e)

AlpDevHalt (deviceid) ;

\} 